\title{
Antioxidant enzymes in the human iris: an immunogold study
}

George Evelyn Marshall

\begin{abstract}
Aims-To determine the nature and extent of the antioxidant enzyme system in the human iris.

Methods-The fine structural distribution of five antioxidant enzymes (acidic, neutral, and basic glutathione S-transferase (GST), $\mathrm{Cu} / \mathrm{Zn}$ superoxide dismutase $(\mathrm{Cu} / \mathrm{Zn}$ SOD), and glutathione peroxidase) was determined by immunogold labelling of ultrathin sections of tissue from six eyes appropriately fixed for immunocytochemistry and embedded in LR white resin.

Results-Both Cu/Zn SOD and acidic GST were localised to all constituent cells of the iris. Glutathione peroxidase and basic GST were localised to erythrocytes alone, and labelling for neutral GST was absent.

Conclusions-Dense labelling for acidic GST could be linked with the previously documented presence of large quantities of polyunsaturated fatty acids in the iris and/or the presence of xenobiotic substances in the aqueous humour.

(Br f Ophthalmol 1997;81:314-318)
\end{abstract}

Iris tissue is a potential site of free radical damage as it is rich in polyunsaturated fatty acids $^{1}$ that are especially susceptible to peroxidation. $^{2-4}$ Information currently available, although limited, supports the hypothesis that antioxidant enzymes are present in the iris in significant quantities. In a light microscopical study of the distribution of $\mathrm{Cu} / \mathrm{Zn}$ superoxide dismutase $(\mathrm{Cu} / \mathrm{Zn}$ SOD) in the rat eye, staining was noted over the posterior pigmented epithelium of the iris..$^{5}$ In addition, biochemical measurement of glutathione peroxidase activity in the rabbit eye indicated that iris and ciliary body had the highest activity among the intraocular tissues examined. ${ }^{6}$ In order to expand an understanding of antioxidant systems, the fine structural distribution of five of the principal antioxidant enzymes was mapped out in the human iris, using antibodies against $\mathrm{Cu} / \mathrm{Zn} \mathrm{SOD}$, glutathione peroxidase, and three forms of human glutathione S-transferase (acidic, basic, and neutral). Immunoelectron microscopy was the technique of choice in the present investigation because it provides detail of the fine structural localisation of the above enzymes.

Materials and methods

Ostensibly normal iris tissue was obtained from six freshly enucleated eyes and the indications for enucleation are shown in Table 1 . The age range of the patients was 2-75 years (Table 1). Fixation and plastic embedding of iris tissue for immunocytochemical studies is described by Konstas et al. ${ }^{7}$ Conventional light and electron microscopy confirmed that the tissue was normal. The immunocytochemical procedure is also described elsewhere. ${ }^{8}$ All primary antibodies were supplied by Biogenesis Ltd (Poole).Those antibodies against the three forms of glutathione S-transferase (alpha, mu, and pi) were rabbit polyclonals and the others were sheep polyclonals. Goat anti-rabbit and donkey anti-sheep secondary immunogold conjugates $(10 \mathrm{~nm})$ were supplied by British Biocell International (Cardiff). Normal goat and rabbit serum negative controls (Sigma) were concurrently conducted at the same dilutions. Omission of the primary antibody was also used as a negative control.

\section{Results}

Tissue from a wide age range of patients was chosen to ensure that the present findings were not a feature of any particular age group. As fixation is the principal factor of tissue processing that affects immunolabelling intensity, strenuous attempts were made to reduce variation in the fixation regime. Thus, fixation was always conducted for 2 hours in $4 \%$ paraformaldehyde with the addition of low concentrations of glutaraldehyde (Table 1). Although phosphate buffered fixation produced better ultrastructural preservation and immunolabelling density than cacodylate buffered fixation, this disparity was eliminated with the addition of $2-6 \%$ sucrose to the cacodylate buffer. It should be stressed that the effect of this form of tissue manipulation produces images which are not encountered in conventional electron microscopy - that is, the illustrations in the present paper appear in 'reversed contrast'. This is due to the omission of osmium tetroxide postfixation which, although standard for conventional transmission electron microscopy, is inap-

Table 1 Details of patients and fixatives used

\begin{tabular}{|c|c|c|c|c|}
\hline Case & Age & Sex & $\begin{array}{l}\text { Fixative } \\
(4 \% P F A \\
\text { plus \% GA) }\end{array}$ & $\begin{array}{l}\text { Indication for } \\
\text { enucleation }\end{array}$ \\
\hline 1 & 2 & $M$ & 0.25 & Retinoblastoma \\
\hline 2 & 9 & $M$ & 0.5 & Injury \\
\hline 3 & 35 & $\mathrm{~F}$ & 0.5 & Orbital tumour \\
\hline 4 & 51 & $\mathrm{~F}$ & 0.2 & Orbital tumour \\
\hline 5 & 63 & $\mathrm{~F}$ & 0.5 & $\begin{array}{l}\text { Choroidal } \\
\text { melanoma }\end{array}$ \\
\hline 6 & 75 & F & 0.2 & $\begin{array}{r}\text { Ciliary body } \\
\text { melanoma }\end{array}$ \\
\hline
\end{tabular}

PFA = paraformaldehyde GA = glutaraldehyde. 

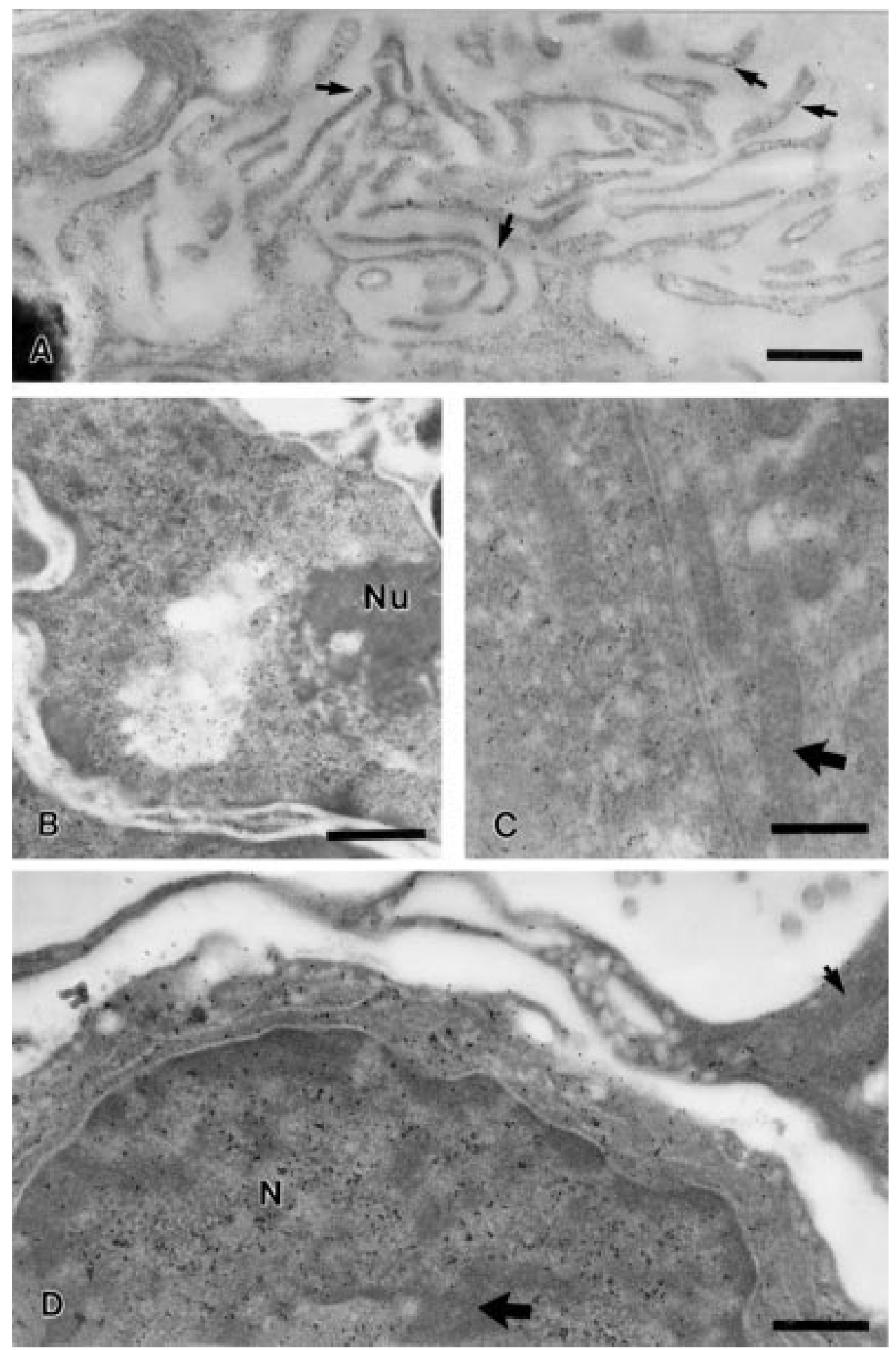

Figure 1 Immunogold labelling for acidic glutathione S-transferase. (A) Immunogold particles are preferentially located over the cell membrane (arrows) of microvillar basal infoldings of the posterior pigmented epithelium. (B) Nucleus of
posterior pigmented epithelial cell exhibits intense labelling; the nucleolus $(\mathrm{Nu})$ is free of label. $(\mathrm{C})$ Cytosol of basal myoportion (dilator muscle) of anterior pigmented myoepithelium. Mitochondria (thick arrows) are devoid of immunogold particles. (D) Densely labelled 'fibrocyte' in iris stroma. Labelling of the nucleus ( $N$ ) is largely confined to the

non-condensed chromatin regions and is absent from mitochondria (small arrow). A region of condensed nuclear chromatin is indicated by large arrow. (All bars $=0.5 \mu \mathrm{m}$.)

propriate for immunocytochemical studies. Thus, biological membranes that have a dark contrast with osmium tetroxide staining, appear here as white lines. The significant findings were as follows: (i) both acidic glutathione S-transferase (Fig 1) and copper/ zinc superoxide dismutase (Fig 2) were located within the cellular components of the iris, (ii) basic glutathione S-transferase and glutathione peroxidase were localised only to erythrocytes 
within the iris vasculature, and (iii) no convincing labelling was observed for neutral glutathione S-transferase.

ACIDIC GLUTATHIONE S-TRANSFERASE-PI

Labelling for acidic glutathione S-transferase-pi (acidic GST) was intense in both the nuclei and cytosol of the posterior pigmented epithelium (Fig 1A, B), the anterior pigmented myoepithelium (Fig 1C), fibrocytes, and melanocytes in both the iris stroma and anterior border layer (Fig 1D). No label was present over extracellular matrix components, such as the basement membranes of the posterior (Fig 1A) and anterior pigmented epithelium, striated collagen fibrils of the stroma, and basement membranes of the iris vasculature. Although cells of the posterior pigment epithelium exhibited rarefaction of their cytoplasm, such rarefaction was not evident in the microvillar basal infoldings or within the nuclei of the cells. Perhaps the most striking feature of immunogold labelling for acidic GST was the preferential localisation of immunogold particles to the cell membrane lining microvillar basal infoldings of the posterior pigmented epithelium (Fig 1A). Dense labelling for acidic GST was present within the cell nuclei. In contrast with the rest of the nucleus, the nucleolus was free of immunogold particles (Fig 1B). Pigment granules were also free of label. No other organelles were observed in the posterior pigment epithelium. Acidic GST was present in the cytosol of the basal and apical regions of the anterior pigmented myoepithelium (Fig 1C). Mitochondria, present in the basal myoportion, were free of label (Fig 1C). Nuclei of the anterior pigmented myoepithelium were not so densely labelled as the nuclei of the posterior pigmented epithelium. Considerable variation in the density of labelling was noted in what were classed as stromal fibrocytes. In most 'fibrocytes' labelling was as intense as that seen in the posterior pigmented epithelium (Fig 1D) and was confined to the nucleus and cytosol, being absent from mitochondria, the lumina of rough endoplasmic reticulum, and nuclear envelope (Fig 1D). Immunogold particles within the nucleus were largely confined to areas of granular nuclear material, being far less prevalent over the more electron dense homogeneous heterochromatin deposits that tended to pack along the inside of the nuclear envelope (Fig 1D). Stromal melanocytes exhibited a similar intensity and pattern of labelling. A minority of 'fibrocytes' were virtually free of immunogold particles. It was not possible, by ultrastructural examination, to differentiate between fibrocytes and some cell types of the immune system, such as MHC class II positive dendritic cells which are resident in the iris. ${ }^{9}$ Labelling was absent from mast cells which were identified by means of their ultrastructural hallmark of 'bearpaw prints'. Some labelling was also observed over vascular supporting cells and the nuclei of vascular endothelial cells. Few immunogold particles were present over the cytoplasm of the vascular endothelial cells, or over the plasma and erythrocytes within the vascular lumen.
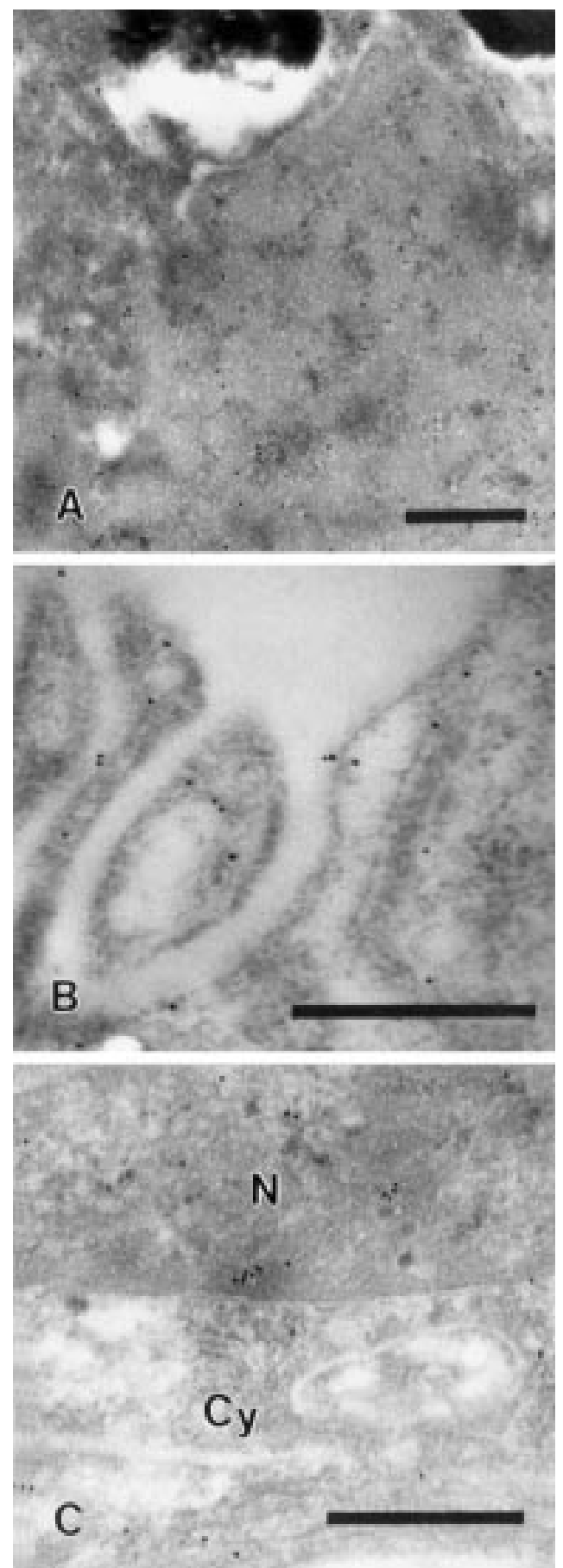

Figure 2 Immunogold localisation for $\mathrm{Cu} / \mathrm{Zn}$ superoxide dismutase. (A) Immunogold particles are localised to the nucleus of a posterior pigmented epithelial cell. The degree of labelling is significantly less than that seen with acidic glutathione S-transferase (GST) (compare with Fig 1B). (B) Microvillar basal infoldings of posterior pigmented epithelium. Unlike acidic GST (Fig 1A) no preferential labelling of the cell membrane is evident. (C) Immunogold particles are present over the nucleus $(N)$ and cytoplasm (Cy) of anterior pigmented myoepithelium.

Fibrocytes (Fig 1D) and melanocytes of the anterior border layer exhibited a similar pattern and density of labelling as the fibrocytes and melanocytes in the iris stroma.

CU/ZN SUPEROXIDE DISMUTASE

The distribution of label for Cu/Zn SOD (Fig 2) was similar to that of acidic GST (Fig 1), but 


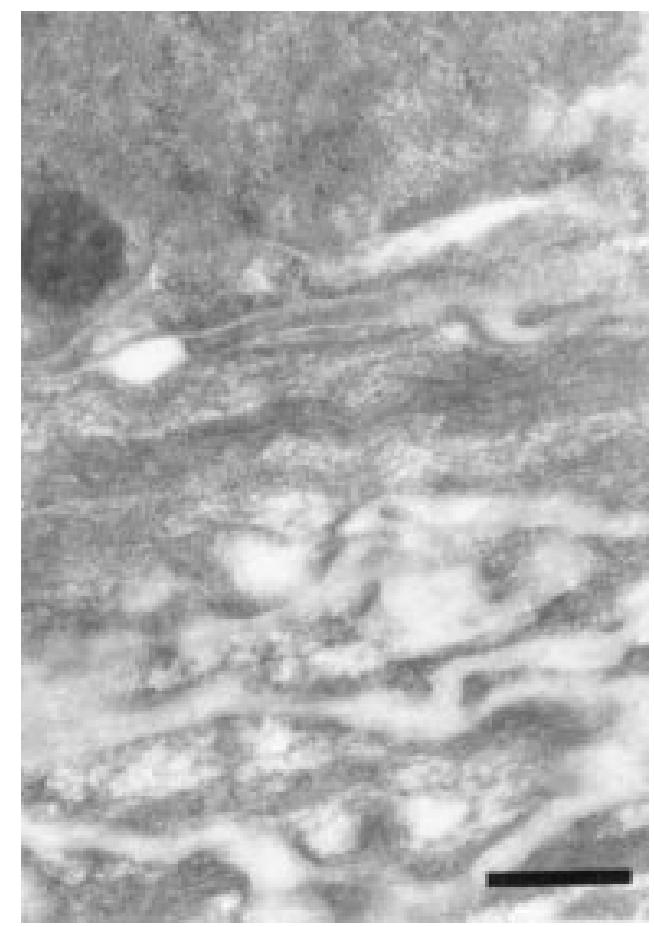

Figure 3 Basal myoportion (dilator muscle) of anterior pigmented myoepithelium is free of label in this donkey anti-sheep immunogold negative control.

the intensity of labelling was significantly less. Positive labelling was present in both the nucleus and cytoplasm of cells in the posterior pigmented epithelium (Fig 2A, B), the anterior pigmented myoepithelium (Fig 2C), fibrocytes, and melanocytes of the stroma and anterior border layer. Mitochondria, endoplasmic reticulum, pigment granules, and nuclear heterochromatin were free of label. Microvillar basal infoldings of the posterior pigmented epithelium exhibited positive labelling (Fig 2B). However, unlike acidic GST, no preferential labelling of the cell membrane lining these infoldings was evident (Fig 2B).

CONTROLS

Immunogold labelling was sparse when the primary antibody was omitted in both the goat anti-rabbit and donkey anti-sheep gold controls (Fig 3). Labelling was non-specific in the normal rabbit serum and normal sheep serum negative controls. Non-specific labelling was greater in normal rabbit serum controls than in normal sheep serum controls.

\section{Discussion}

Although the labelling intensity for acidic GST was greater than that of $\mathrm{Cu} / \mathrm{Zn} \mathrm{SOD}$, this does not necessarily imply that acidic GST is the more abundant of the two enzymes but may reflect dissimilar sensitivities of the antigenic epitopes to tissue processing (particularly fixation) or different avidities of the antibodies to their respective epitopes. Such factors, however, would not influence the specific localisation within constituent cells, such as the preferential labelling of the cell membrane in basal infoldings of the posterior pigmented epithelium with acidic GST antibodies and the absence of such labelling with $\mathrm{Cu} / \mathrm{Zn}$ SOD. Inequality of labelling between the three isoenzymes of GST (acidic, basic, and neutral) has also been observed in other human tissues. ${ }^{10}$ The abundance of acidic GST in iris tissue could be ascribed to the high content of polyunsaturated fatty acids (PUFAs) in the tissue, ${ }^{1}$ as glutathione $\mathrm{S}$-transferases play a critical role in neutralising lipid hydroperoxides, ${ }^{11}{ }^{12}$ thereby preventing any further damage arising from a chain reaction of peroxidation. ${ }^{13}$ Alternatively, it may be linked to the iris being immersed in a 'bathing fluid', as cells bathed in a specialised extracellular matrix fluid exhibit intense staining for GST. ${ }^{14}$ Examples include Muller cells of the retina which are in contact with the vitreous, ${ }^{14}$ Bergmann cells of the cerebellum, that are bathed in cerebrospinal fluid, ${ }^{15}$ and hair cells of organ of Corti which are bathed in endolymph fluid. ${ }^{16}$ The iris can be added to this list, as it is bathed in aqueous humour and, as the present study has shown, contains an abundance of GST. Since glutathione S-transferases have been shown to function in mammalian tissues as metabolic detoxicants of diverse electrophilic xenobiotics ${ }^{17-19}$ these cell types may require GST to process exogenous toxicants present in the bathing fluid. ${ }^{14}$ Acidic GST has not previously been localised at the ultrastructural level in any human or animal tissue. That acidic GST was located to the cytoplasmic ground substance and not to the organelles within the cytoplasm is therefore a novel feature of the present study. Obviously, the resolution afforded by electron microscopy was required to show the absence of labelling for acidic GST from organelles such as mitochondria and the lumina of rough and smooth endoplasmic reticulum. Another striking feature was the intense labelling of the noncondensed chromatin regions (see Fig1D) and its absence from nucleoli (Fig 1B) and the dense heterochromatin of the nucleus (Fig 1D). This finding is interpreted as an active importation of acidic GST into the nucleus, via the nuclear pores. Its location within the non-condensed chromatin may reflect the vulnerability of less well packaged DNA to free radical damage. Oxidative stress to DNA takes the form of 'strand breaks' and damage to bases and sugars which results from hydroxy radical induced damage or activation of nucleases. ${ }^{20}$ That acidic GST was also located in the nuclei of all cell types is an indication of its function of conjugating reduced glutathione with oxidised DNA. ${ }^{21}$ Perhaps the most striking feature in the distribution of acidic GST was its preferential localisation to the cell membrane lining the basal infoldings of the posterior pigmented epithelium. This feature could be cited as evidence for the importance of acidic GST in detoxifying lipid hydroperoxides since the cell membranes lining the basal infoldings are rich in polyunsaturated fatty acids. ${ }^{22}$ It could equally be used as evidence for a demand for the processing of exogenous toxicants since the basal infoldings of the posterior pigmented cells are bathed in aqueous humour. Several lines of evidence suggest that 
some of the GST isoenzymes play a pivotal role in protecting cells from the consequences of oxidative stress. Hays and Strange ${ }^{21}$ state that an assessment of the importance of these GSTs in humans is difficult at present, because the number of isoenzymes within the alpha and theta class is not yet known and the catalytic preferences of even identified isoforms is not always clear. There is limited information on the ultrastructural distribution of $\mathrm{Cu} / \mathrm{Zn}$ SOD in animal tissues. ${ }^{1123}$ Biochemical and light microscopic studies demonstrated a large concentration in the iris and ciliary body ${ }^{24}$ with localisation to the posterior pigment epithelial layer of the iris. ${ }^{5}$ Immunogold labelling for $\mathrm{Cu} / \mathrm{Zn}$ SOD was absent from mitochondria in the present study. This feature was also noted in enterocytes of the avian intestine ${ }^{25}$ and is to be expected, as mitochondria possess a different form of SOD (MnSOD) ${ }^{26-28}$ The dismutation of superoxide anions by $\mathrm{Cu} / \mathrm{Zn}$ SOD results in the production of hydrogen peroxide which, in turn, is neutralised either by glutathione peroxidase or catalase. The lack of glutathione peroxidase in iris tissue is compensated for by the presence of catalase whose activity has been detected in human iris $^{29}$ and which has been immunohistochemically demonstrated in the rat iris. ${ }^{30}$ In summary, the use of immunogold labelling has revealed the subtlety of antioxidant enzyme distribution in the iris. This affords a route for further investigation of light induced damage to the anterior segment tissues and the potential for pharmacological protection.

The author is indebted to Professor W R Lee for comments on the manuscript and to Mrs Sophia Cameron for technical assistance. This work was supported by the Sir Jules Thorn Charitable Trust.

1 Zhang Y, Yousufzai SYK, Abdel-Latif AA. Comparative studies on fatty acid composition and phospholipases A2 and $C$ reactivities in rabbit and bovine iris-ciliary body. Exp Eye Res 1993;56:151-5.

2 Rice-Evans CA, Diplock AT. Current status of antioxidant therapy. Free Radic Biol Med 1993;15:77-96.

3 Floyd RA, Carney JM. Free radical damage to protein and DNA: mechanisms involved and relevant observations on brain undergoing oxidative stress. Ann Neurol 1992;32 S22-7.

4 Cheeseman KH. Mechanisms and effects of lipid peroxidation. Mol Aspects Med 1993;14:191-7.

5 Rao NA, Thaete LG, Delmage JM, Sevanian A. Superoxide dismutase in ocular structures. Invest Ophthalmol Vis Sci 1985;26:1778-81.

6 Bhuyan KC, Bhuyan DK. Regulation of hydrogen peroxide in eye humors: effect of 3-amino-IH-1,2,4-triazole on catalase and glutathione peroxidase of rabbit eye. Biochim Biolase and glutathione peroxidase
phys Acta 1977;497:641-56.

7 Konstas AG, Marshall GE, Lee WR. Immunocytochemical localisation of collagens (I-V) in the human iris. Graefes localisation of collagens (I-V) in the hum
Arch Clin Exp Ophthalmol 1990;228:180-6.

8 Marshall GE, Konstas AGP, Lee WR. Collagens in the aged human macular sclera. Curr Eye Res 1993;12:143-53.

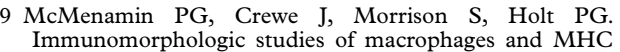
class II-positive dendritic cells in the iris and ciliary body of the rat, mouse and human eye. Invest Ophthalmol Vis Sci 1994;35:3234-50.

10 Hayes PC, Harrison DJ, Boucher IAD, McLellan LI, Hayes JD. Cytosolic and microsomal glutathione S-transferase isoenzymes in normal human liver and intestinal epithelium. Gut 1989;30:854-9.

11 Muse KE, Oberley TD, Sempf JM, Oberley LW. Immunolocalization of antioxidant enzymes in adult hamster kidney. Histochem f 1994; 26:734-53.

12 Jensson H, Guthenberg C, Alin P, Mannervik B. Rat glutathione transferase 8-8, an enzyme efficiently detoxifying 4-hydroxy- alk-2-enals. FEBS Lett 1986;203:207-9.

13 Attala LR, Sevanian A, Rao NA. Immunohistochemical localization of glutathione peroxidase in ocular tissue. Curr Eye Res 1988;7:1023-7.

14 McGuire S, Daggett D, Bostad E, Schroeder S, Siegel F, Kornguth S. Cellular localization of glutathione S-transferases in retinas of control and lead-treated rats. Invest Ophthalmol Vis Sci 1996;37:833-42.

15 Johnson JA, El Barbary A, Kornguth SE, Brugge JF, Siegel FL. Glutathione $S$ transferase iso-enzymes in rat brain neurons and glia. $\mathcal{f}$ Neuroscience 1993;13:2013-23.

neurons and glia. F Neuroscience 1993;13:2013-23.
16 El Barbary A, Altschuler RA, Schacht J. Glutathione S-transferases in the organ of Corti of the rat: enzymatic activity, subunit composition and immunohistochemical localization. Hearing Res 1993;71:80-90.

17 Ketterer B, Meyer DJ, Clark AG. Soluble glutathione transferase isoenzymes. In: Sies $\mathrm{H}$, Ketterer B, eds. Glutathione conjugation: mechanisms and biological significance. London: Academic Press, 1988:73-135.

18 Mannervik B, Alin P, Guthenberg C, Jensson H, Tahir MK, Warholm M, et al. Identification of three classes of cytosolic glutathione transferase common to several mammalian species:correlation between structural data and enzymatic properties. Proc Natl Acad Sci USA 1985;82:7202-6.

19 Vos RME, Van Bladeren PJ. Glutathione S transferases in relation to their role in the biotransformation of xenobiotics. Chem Biol Interact 1990;75:241-65.

20 Halliwell B, Aruoma OI. DNA damage by oxygen-derived species. Its mechanism and measurement in mammalian systems. FEBS Lett 1991;281:9-19.

21 Hayes JD, Strange RC. Potential contribution of the glutathione S-transferase supergene family to resistance to oxidative stress. Free Radic Res 1995;22:193-207.

22 Ishimoto S, Wu G-S, Hayashi S, Zhang J, Rao NA. Free radical tissue damages in the anterior segment of the eye in experimental autoimmune uveitis. Invest Ophthalmol Vis Sci 1996;37:630-6.

23 Coursin DB, Cihla HP, Oberley TD, Oberley LW. Immunolocalization of anti-oxidant enzymes and isoen-
zymes of glutathione S-transferase in normal rat lung. $\mathrm{Am}$ zymes of glutathione S-trans

24 Bhuyan KC, Bhuyan DK. Superoxide dismutase of the eye. Relative functions of superoxide dismutase and catalase in protecting the ocular lens from oxidative damage. Biochim Biophys Acta 1978;542:28-38.

25 Davis WL, Matthews JL, Shibata K, Kipnis M, Farmer GR, Cortinas E, et al. The immunocytochemical localization of superoxide dismutase in the enterocytes of the avian intestine: the effect of vitamin D3. Histochem $\mathcal{f} 1989 ; 21$ : 194-202.

26 Oberley TD, Coursin DB, Cihla HP, Oberley LW, El-Sayyad N, Ho, Y. Immunolocalization of manganese El-Sayyad $\mathrm{N}, \mathrm{Ho}, \mathrm{Y}$. Immunolocalization of manganese
superoxide dismutase in normal and transgenic mice superoxide dismutase in normal and transgenic mice 79 .

27 Fridovich I. The biology of oxygen radicals: the superoxide radical is an agent of oxygen toxicity-superoxide dismutases provide an important defense. Science 1978;201: 875-80.

28 Weisiger RA, Fridovich I. Mitochondrial superoxide dismutase. Site of synthesis and intramitochondrial localization. F Biol Chem 1973;248:3582-92.

29 Liles MR, Newsome DA, Oliver PD. Antioxidant enzymes in aging human retinal pigment epithelium. Arch Ophthalmol 1991; 109:1285-8.

30 Atalla L, Fernandez MA, Rao NA. Immunohistochemical localization of catalase in ocular tissues. Curr Eye Res 1987; 6:1181-7 